\title{
ARTICLE OPEN Phase-field model of pitting corrosion kinetics in metallic materials
}

\author{
Talha Qasim Ansari ${ }^{1}$, Zhihua Xiao ${ }^{1}$, Shenyang $\mathrm{Hu}^{2}$, Yulan $\mathrm{Li}^{2}$, Jing-Li Luo ${ }^{3}$ and San-Qiang Shi ${ }^{1}$
}

Pitting corrosion is one of the most destructive forms of corrosion that can lead to catastrophic failure of structures. This study presents a thermodynamically consistent phase field model for the quantitative prediction of the pitting corrosion kinetics in metallic materials. An order parameter is introduced to represent the local physical state of the metal within a metal-electrolyte system. The free energy of the system is described in terms of its metal ion concentration and the order parameter. Both the ion transport in the electrolyte and the electrochemical reactions at the electrolyte/metal interface are explicitly taken into consideration. The temporal evolution of ion concentration profile and the order parameter field is driven by the reduction in the total free energy of the system and is obtained by numerically solving the governing equations. A calibration study is performed to couple the kinetic interface parameter with the corrosion current density to obtain a direct relationship between overpotential and the kinetic interface parameter. The phase field model is validated against the experimental results, and several examples are presented for applications of the phase-field model to understand the corrosion behavior of closely located pits, stressed material, ceramic particles-reinforced steel, and their crystallographic orientation dependence.

npj Computational Materials (2018)4:38; doi:10.1038/s41524-018-0089-4

\section{INTRODUCTION}

Corrosion is the gradual destruction of materials (usually metallic materials) via chemical and/or electrochemical reaction with their environment. It costs about $3.1 \%$ of the gross domestic product (GDP) in the United States, which is much more than the cost of all natural disasters combined. Localized corrosion, such as pitting corrosion, is one of the most destructive forms of corrosion; it leads to the catastrophic failure of structures and raises both human safety and financial concerns. ${ }^{1-3}$ Pitting corrosion of stainless steel usually occurs in two different stages: (1) pit initiation from passive film breakage ${ }^{4-6}$ and (2) pit growth. ${ }^{2,3,7-12}$ In this study, we focused on the development of a phase-field modeling capability to study pit growth by considering both anodic and cathodic reactions.

In the past few decades, great efforts have been made to develop numerical models for pitting corrosion. The moving interface and the electrical double layer at the metal/electrolyte interface are the two challenging problems faced by most of these numerical models. These numerical models can be divided according to the method in which a moving interface is incorporated in their models. Several steady state ${ }^{9,10,13-18}$ and transient state ${ }^{19-28}$ models have been developed over the years that did not allow for changes in the shape and dimensions of the pits/crevices as corrosion proceeds.

Recent advances in numerical techniques, such as the finite element method, the finite volume method, the arbitrary Lagrangian-Eulerian method, the mesh-free method, and the level set method have encouraged researchers to model the evolving morphology of the pits with a moving interface. Most of these modeling efforts have used a sharp interface to represent the corroding surface, which requires the matching mesh at each time step, ${ }^{11,29}$ thus increasing the errors associated with the violation of mass conservation laws and increasing the computation cost. The finite volume method models overcome this problem by creating a matching mesh as a function of the concentration of ions, but they are still unable to model complex microstructures. ${ }^{12,30,31}$ A mesh-free method, the peridynamic model, has been implemented to model pitting corrosion, but it only considered electrochemical reactions without considering the ionic transport in the electrolyte. ${ }^{7}$

Over the past three decades, the phase field (PF) method has emerged as a powerful simulation tool for modeling of microstructure evolution. PF models study the phase transformation by defining the system's free energy, and the system's microstructure evolution is predicted by free energy minimization. The PF approach has been extensively applied to many materials processes, such as solidification, dendrite growth, solute diffusion and segregation, phase transformation, electrochemical deposition, dislocation dynamics, crack propagation, void formation and migration, gas bubble evolution, and electrochemical processes. ${ }^{32}$

PF models assume a diffusive interface at the phase boundaries rather than a sharp one, which makes the mathematical functions continuous at the interface. A few recent attempts have been made to use the PF method to model pitting corrosion and stress corrosion cracking without the consideration of cathodic reactions, ionic transport and in particular the dependence of overpotential on metal ion concentration in the electrolyte. ${ }^{33,34}$ In reality, the transport of ionic species in the electrolyte often plays a very important role in diffusion controlled corrosion processes, and the effects of these ionic species must be incorporated to model the process adequately. In this study, a PF method is used to model pitting corrosion by considering both

\footnotetext{
${ }^{1}$ Department of Mechanical Engineering, The Hong Kong Polytechnic University, 11 Yuk Choi Road, Hung Hom, Kowloon, Hong Kong; ${ }^{2}$ Pacific Northwest National Laboratory, Richland, WA 99352, USA and ${ }^{3}$ Department of Chemical and Materials Engineering, University of Alberta, Edmonton, AB T6G 2R3, Canada Correspondence: S-Q. Shi (mmsqshi@polyu.edu.hk)
}

Received: 20 November 2017 Revised: 13 June 2018 Accepted: 18 June 2018

Published online: 24 July 2018 
anodic and cathodic reactions, transport of ionic species and the dependence of overpotential on metal ion concentration in the electrolyte.

This paper is organized as follows. Firstly, we describe the system and the electrochemical reactions considered in this work followed by the construction of PF model. The total free energy of this PF model consists of three parts: bulk free energy, gradient energy and electrostatic energy. We used the KKS model ${ }^{35}$ to construct the bulk free energy and the interfacial energy. Secondly, we developed the governing equations which comprise of mass diffusion, electromigration, and chemical reaction terms, whereas the interface conditions are incorporated by introducing an order parameter that defines the system's physical state at each material point. Thirdly, a study is included to couple the kinetic interface parameter and the system's total overpotential. Fourthly, the PF model is validated against the experimental results and previous numerical models. Lastly, several case studies are presented to demonstrate the strength of this proposed PF model.

\section{RESULTS AND DISCUSSION}

The system and electrochemical reactions considered

The system studied consists of stainless steel 304 (SS304) in dilute salt water (Fig. 1). It is assumed that new passive film will not form in this system. We will consider the effects of passive film formation in a future study. In this model, the following electrochemical reactions and kinetics are considered.

For the oxidation of main metal alloy elements in SS304,

$$
\begin{aligned}
& \mathrm{Fe} \rightarrow \mathrm{Fe}^{+2}+2 e^{-} \\
& \mathrm{Ni} \rightarrow \mathrm{Ni}^{+2}+2 e^{-} \\
& \mathrm{Cr} \rightarrow \mathrm{Cr}^{+3}+3 e^{-}
\end{aligned}
$$

In the following, $M_{e}$ is used to represent the effective metal in SS304 with an average charge number of $z_{1}$. The material properties of SS304 such as molar concentration in solid phase $\left(c_{\text {solid }}=143 \mathrm{~mol} / \mathrm{L}\right){ }^{12}$ saturation concentration in the electrolyte $\left(c_{\text {sat }}=5.1 \mathrm{~mol} / \mathrm{L}\right){ }^{12}$ effective diffusion coefficient $\left(D_{1}=8.5 \times 10^{-10}\right.$ $\left.\mathrm{m}^{2} / \mathrm{s}\right){ }^{12}$ and the average charge number $\left(z_{1}=2.19\right)$ based on $\mathrm{Fe}$, $\mathrm{Ni}, \mathrm{Cr}$, and their mole fractions within the alloy (taken from ref. ${ }^{12}$ ). The above reactions can then be simplified to

$$
M_{e} \rightarrow M_{e}^{z_{1}}+z_{1} e^{-}
$$

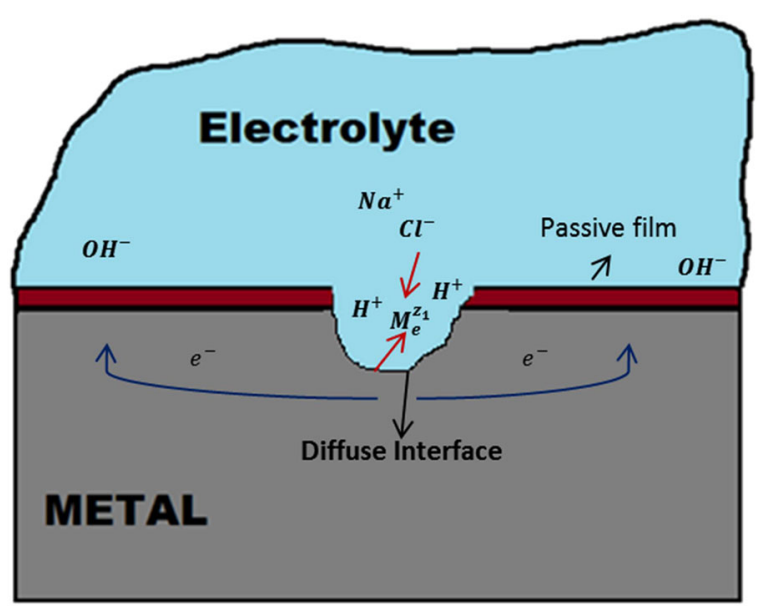

Fig. 1 Schematic of the chemical reactions that occur during the pitting corrosion process
The anodic dissolution of the metal is assumed to follow Butler-Volmer equation,

$$
i_{a}=i_{0}\left[\exp \left(\frac{a_{a} z_{1} F \varphi_{s, o}}{R_{g} T}\right)-\exp \left(-\frac{a_{c} z_{1} F \varphi_{s, o}}{R_{g} T}\right)\right]
$$

where $F$ is the Faraday constant, $R_{\mathrm{g}}$ is the gas constant, $T$ is the temperature, $\varphi_{\mathrm{s}, \mathrm{o}}$ is the polarization overpotential, $i_{\mathrm{o}}$ is the exchange current density, $a_{\mathrm{a}}$ is the anodic charge transfer coefficient, $a_{\mathrm{c}}$ is the cathodic charge coefficient $\left(a_{\mathrm{c}}=1-a_{\mathrm{a}}\right)$. The values of the above mentioned parameters are reported in Table 1s (supplementary material).

For the hydrogen discharge reaction in Eq. (3), the corresponding rate is given in Eq. (4)

$H^{+}+e^{-} \rightarrow \frac{1}{2} H_{2}$

$J_{5}=J_{50}\left[H^{+}\right] \exp \left(\frac{a_{5} F}{R_{g} T} \varphi_{s, o}\right)$

For reduction of water (Eq. (5)), the corresponding rate is given in Eq. (6)

$\mathrm{H}_{2} \mathrm{O}+e^{-} \rightarrow \mathrm{H}+\mathrm{OH}^{-}$

$J_{6}=J_{60} \exp \left(\frac{a_{6} F}{R_{g} T} \varphi_{s, 0}\right)$

Experimental values of $i_{0}, J_{50}, J_{60}, a_{a}, a_{5}$, and $a_{6}$ are given in Table 1s.

In this work, the following two reactions in the electrolyte are considered

$\mathrm{M}_{e}^{z_{1}}+\mathrm{H}_{2} \mathrm{O} \rightleftharpoons \mathrm{M}_{e} \mathrm{OH}^{z_{1}-1}+H^{+}$

$\mathrm{H}_{2} \mathrm{O} \rightleftharpoons \mathrm{OH}^{-}+\mathrm{H}^{+}$

The equilibrium constants of reactions in Eqs. (7) and (8) are defined as $K_{1}$ and $K_{2}$, respectively.

$K_{1}=\frac{k_{1 f}}{k_{1 b}}, K_{2}=\frac{k_{2 f}}{k_{2 b}}$

where $k_{1 \mathrm{f}}, k_{1 \mathrm{~b}}, k_{2 \mathrm{f}}$, and $k_{2 \mathrm{~b}}$ are the forward and backward reaction rates. Therefore, a total of six ion species are considered in this model,

$M_{e}^{z_{1}}=c_{1} ; M_{e} \mathrm{OH}^{z_{2}}=c_{2} ; \mathrm{Cl}^{z_{3}}=c_{3} ; \mathrm{Na}^{z_{4}}=c_{4} ; H^{z_{5}}=c_{5} ; \mathrm{OH}^{z_{6}}=c_{6}$

where $z_{i}(i=1,2, \ldots \ldots 6)$ are the charge numbers of the respective species (their values are given in Table $2 \mathrm{~s})$, and $c_{i}(i=1,2, \ldots \ldots 6)$ are the normalized concentrations of the respective species. The normalized concentration $c_{i}$ is determined by $C_{i}=C_{i} / C_{\text {solid }}$ for $i=$ $1,2, \ldots, 6$, where $C_{i}$ represents the molar concentration of ionic species. The constants $K_{1}$ and $K_{2}$ can also be expressed as a function of $C_{i}$.

$K_{1}=C_{2} C_{5} / C_{1}, K_{2}=C_{5} C_{6}$

The phase field model for corrosion

The surface of the metal is normally covered with the passive film; however, a partial breakdown in the film can occur, which may initiate pits like the one illustrated in Fig. 1. The model consists of two phases: the solid phase $M_{e}$ (i.e., the metal part) and the liquid phase (i.e., the electrolyte part). The driving force for metal corrosion and microstructure evolution is from the minimization of the system's total free energy, which usually consists of bulk free energy $E_{\mathrm{b}}$, interface energy $E_{\mathrm{i}}$, and long-range interaction energies such as elastic strain energy $E_{\mathrm{s}}$ and electrostatic energy $E_{\mathrm{e}}{ }^{36,37}$ The system's total energy can be expressed as

$E=E_{b}+E_{i}+E_{s}+E_{e}$ 
The inclusion of elastic and/or plastic deformation in the model is completely feasible because it has been done in other systems. ${ }^{38-40}$ It can be even necessary to include the strain energy term if a volumetric non-compatible passive film develops during corrosion. Because the formation of a passive film will not be considered in the first stage of this work, for simplicity, the elastic strain energy is not considered here. In a later section, the effect of applied or residual stress on pitting will be studied using the concept of overpotential rather than strain energy. Now we have,

$E=E_{b}+E_{i}+E_{e}$

$E=\int\left[f_{b}\left(c_{1}, \eta\right)+\frac{a_{u}}{2}(\nabla \eta)^{2}+z_{1} F C_{1} \varphi\right] d V$

where $f_{\mathrm{b}}\left(c_{1}, \eta\right)$, derived in the next section, is the local bulk free energy density, which is a function of the normalized concentration of the ionic species $c_{1}$ and order parameter $\eta$; the second term in Eq. (11) represents the gradient energy density that contributes to the interfacial energy, in which $a_{u}$ is the gradient energy coefficient, which is related to physical parameters in a later section; and the third term in Eq. (11) represents the electrostatic energy density where $C_{1}$ is the molar concentration of metal ion and $\varphi$ is the electrostatic potential.

Bulk free energy density

To determine the bulk free energy density $f_{\mathrm{b}}\left(c_{1}, \eta\right)$, we use the model proposed by Kim et al. for binary alloys. ${ }^{35}$ We chose KKS model because the model has less limitations on the interface thickness as compared to some other models such as model presented by the model by Wheeler et al. ${ }^{41}$ The detailed derivations of all functions in the KKS model were skipped here and readers are referred to the original paper. ${ }^{35}$ In KKS model, the model parameters can be analytically determined by material properties and experimental conditions for the concerned system. In KKS model, at each point, the material is regarded as a mixture of two coexisting phases, and a local equilibrium between the two phases is always assumed:

$c_{1}=h(\eta) c_{s}+[1-h(\eta)] c_{l}$

$\partial f_{s}\left(c_{s}\right) / \partial c_{s}=\partial f_{l}\left(c_{l}\right) / \partial c_{l}$

where $c_{\mathrm{s}}$ and $c_{\mathrm{l}}$ represent the normalized concentrations of the solid and liquid phases, respectively; $h(\eta)$ is a monotonously varying function from $h(0)=0$ to $h(1)=1$. In this study, it is assumed that $h(\eta)=\eta^{2}(-2 \eta+3)$. In Eq. (13), the free energy densities of the solid and liquid phases are expressed as $f_{s}\left(c_{s}\right)$ and $f_{1}\left(c_{1}\right)$, respectively. Because the concentration is considered to be a mixture of solid and liquid phases at each point, by following the same argument, the bulk free energy density of the solid and liquid phases are expressed in a similar manner,

$f_{b}\left(c_{1}, \eta\right)=h(\eta) f_{s}\left(c_{s}\right)+[1-h(\eta)] f_{l}\left(c_{l}\right)+w g(\eta)$

This is a double well potential in the energy space. The height of the double well potential is $w$, and $g(\eta)=\eta^{2}(1-\eta)^{2}$. This expression has two minima at $\eta=0$ and $\eta=1$, which represent the electrolyte phase and the solid phase, respectively.

For this system, $f_{\mathrm{s}}\left(c_{\mathrm{s}}\right)$ and $f_{\mathrm{l}}\left(c_{\mathrm{l}}\right)$ can reasonably be considered as parabolic functions. ${ }^{42}$

$f_{s}\left(c_{s}\right)=A\left(c_{s}-c_{e q, s}\right)^{2}$

$f_{l}\left(c_{l}\right)=A\left(c_{l}-c_{e q, l}\right)^{2}$

where $C_{\text {eq, }}=1$ and $C_{\text {eq, }}=C_{\text {sat }} / C_{\text {solid }}$ are the normalized equilibrium concentrations of the solid and liquid phases, respectively. The temperature-dependent free energy density proportionality constant $A$ is considered to be equal for both the liquid and solid phases. Its value is calculated in such a manner that the driving force for the metal corrosion in the approximated resulting system is quite close to that of the original thermodynamic system. ${ }^{42}$

The evolution of phase order parameter $\eta$ and metal ion concentration $c_{1}$ in time and space are assumed to obey the Ginzburg-Landau (also known as Allen-Cahn) ${ }^{43}$ and Cahn-Hilliard $^{44}$ equations, respectively.

$$
\frac{\partial \eta}{\partial t}=-L \frac{\delta E}{\delta \eta}=L\left[\nabla a_{u} \nabla \eta+h \prime(\eta)\left\{f_{l}\left(c_{l}\right)-f_{s}\left(c_{s}\right)-\left(c_{l}-c_{s}\right) \frac{\partial f_{l}\left(c_{l}\right)}{\partial c_{l}}\right\}-w g_{\prime}(\eta)\right]
$$

$$
\begin{aligned}
\frac{\partial c_{1}}{\partial t}= & \nabla M \nabla \frac{\delta E}{\delta c_{1}}+R_{1} \\
=\nabla & {\left[D_{1}(\eta) \nabla c_{1}\right]+\nabla\left[D_{1}(\eta) h \prime(\eta)\left(c_{l}-c_{s}\right) \nabla \eta\right] } \\
& +\nabla\left[\frac{z_{1} F c_{1} D_{1}(\eta)}{R_{g} T} \nabla \varphi\right] y_{1}(\eta)+R_{1}
\end{aligned}
$$

where $L$ is the kinetic parameter that represents the solid-liquid interface mobility, and $M$ is the mobility of metal ions and expressed as $M=D_{1}(\eta) /\left(\partial^{2} f_{b} / \partial c_{1}^{2}\right)$. In Eq. (18), $R_{1}$ is the source and/or sink term for metal ions due to reaction (Eq. (7)), and it takes the form of $\left(-k_{1 f} c_{1}+k_{1 b} c_{2} c_{5}\right) y_{1}(u)$. The function $y_{1}(\eta)$ defined below is to ensure that reaction (Eq. (7)) occurs only in the electrolyte phase.

$$
\mathrm{y}_{1}(\eta)= \begin{cases}1 ; & \eta \leq 0 \\ & 0<\eta<0.1 \text { (linearly change from } 1 \text { to } 0) \\ 0 ; & \eta \geq 0.1\end{cases}
$$

\section{Conservation of charge}

In this study, we follow Dassault's work rather than following Guyer's model ${ }^{45,46}$ which simplifies the model by removing the need to discretize the double layer at the metal-electrolyte interface. It allows our PF model to simulate the corrosion process from meso- to macro-length scales, as compared to Guyer's model, which was limited to nanoscale. It is also possible to incorporate the effect of the laminar/turbulent flow of the electrolyte on the metal-electrolyte interface in case of moving electrolyte. ${ }^{47}$ Here, the conservation of charge can be expressed as

$$
\frac{\partial \rho_{e}}{\partial t}=\nabla\left\{\sigma_{e}\left[1-y_{1}(\eta)\right] \nabla \varphi\right\}+y_{1}(\eta) F C_{\text {solid }} \sum z_{i} \frac{\partial c_{i}}{\partial t}
$$

where $\rho_{\mathrm{e}}$ is the charge density and $\sigma_{\mathrm{e}}$ is the electrical conductivity of the metal in the solid phase. The function $\left[1-y_{1}(n)\right]$ interpolates the electrical conductivity, $\sigma_{\mathrm{e}}$ in the solid phase to zero in the electrolyte phase, where $y_{1}(\eta)$ is defined in Eq. (19). The time needed for charge accumulation across the interface due to the diffusion of ionic species is much longer than that required to achieve steady-state charge accumulation across the interface, so the conservation of charge in the above system can be considered at a steady state. The relation given in Eq. (20) is reduced to

$0=\nabla\left\{\sigma_{e}\left[1-y_{1}(\eta)\right] \nabla \varphi\right\}+y_{1}(\eta) F C_{\text {solid }} \sum z_{i} \frac{\partial c_{i}}{\partial t}$

\section{Gradient energy coefficient}

The height of the double well potential $w$ and the gradient energy coefficient $a_{u}$ can be related to the interface energy $\varrho$ and the interface thickness $P^{33}$

$\varrho=4 \sqrt{w a_{u}}$

$I=\sqrt{2} a \prime \sqrt{\frac{a_{u}}{w}}$

where $a^{\prime}$ is a constant value determined by the order parameter $u$. If the interface region is defined as $0.05<\eta<0.95$; the value of $a^{\prime}$ is $2.94 .^{35}$ 
Transport equations for other ionic species in the electrolyte

The governing equations of the other five ionic species are the Nernst-Planck equations with chemical reaction terms. They are expressed as

$$
\begin{aligned}
& \frac{\partial c_{2}(\boldsymbol{x}, t)}{\partial \mathrm{t}}=\nabla\left[D_{2}(\eta) \nabla c_{2}\right]+\nabla\left[\frac{z_{2} F c_{2} D_{2}(\eta)}{R_{g} T} \nabla \varphi\right] y_{1}(\eta)+R_{2} \\
& \frac{\partial c_{3}(\boldsymbol{x}, t)}{\partial \mathrm{t}}=\nabla\left[D_{3}(\eta) \nabla c_{3}\right]+\nabla\left[\frac{z_{3} F c_{3} D_{3}(\eta)}{R_{g} T} \nabla \varphi\right] y_{1}(\eta) \\
& \frac{\partial c_{4}(\boldsymbol{x}, t)}{\partial \mathrm{t}}=\nabla\left[D_{4}(\eta) \nabla c_{4}\right]+\nabla\left[\frac{z_{4} F c_{4} D_{4}(\eta)}{R_{g} T} \nabla \varphi\right] y_{1}(\eta) \\
& \frac{\partial c_{5}(\boldsymbol{x}, t)}{\partial \mathrm{t}}=\nabla\left[D_{5}(\eta) \nabla c_{5}\right]+\nabla\left[\frac{z_{5} F c_{5} D_{5}(\eta)}{R_{g} T} \nabla \varphi\right] y_{1}(\eta)+R_{5} \\
& \frac{\partial \mathrm{c}_{6}(\boldsymbol{x}, t)}{\partial \mathrm{t}}=\nabla\left[D_{6}(\eta) \nabla c_{6}\right]+\nabla\left[\frac{z_{6} F c_{6} D_{6}(\eta)}{R_{g} T} \nabla \varphi\right] y_{1}(\eta)+R_{6}
\end{aligned}
$$

where $R_{2}$ is the source/sink term originated from the electrochemical reaction in Eq. (7) which takes the form as $\left[k_{1 f} c_{1}-\right.$ $\left.k_{1 b} C_{2} C_{5}\right] y_{1}(\eta)$. The rates of forward and backward reaction are expressed by $k_{1 f}$ and $k_{1 b}$ respectively. It is assumed that no electrochemical reaction occurs inside the metal part. This is ensured by $y_{1}(\eta)$ defined in Eq. (19). $R_{5}$ and $R_{6}$ are the source/sink terms originated from electrochemical reactions in Eqs. (7) and (8) and take the form as $\left[k_{1 f} c_{1}-k_{1 b} c_{2} c_{5}+k_{2 f}-k_{2 b} c_{5} c_{6}\right] y_{1}(\eta)-$ $\left(\frac{J_{5}}{z_{5} F C_{\text {solid }}}\right) y_{2}(\eta)$ and $\left[k_{2 f}-k_{2 b} c_{5} c_{6}\right] y_{1}(\eta)-\left(\frac{J_{6}}{z_{6} F C_{50 l i d}}\right) y_{2}(\eta)$ respectively. The rates of forward and backward reactions for the hydrolysis of water are represented by $k_{2 f}$ and $k_{2 b}$, respectively. It should be noted that $R_{5}$ and $R_{6}$ have an additional term near the metal-electrolyte interface due to the cathodic reactions considered in Eqs. (3) and (5) where $J_{5}$ and $J_{6}$ are defined in Eqs. (4) and (6) respectively. These reaction terms are multiplied by a step function $y_{2}(\eta)$ to ensure that these reactions only happen in a small region near the metal surface.

$\mathrm{y}_{2}(\eta)= \begin{cases}1 ; & 0.01 \leq \eta<0.05 \\ 0 ; & \eta \geq 0.05 \\ 0 ; & \eta<0.01\end{cases}$

It should also be noted that, in Eqs. (25) and (26) there are no source/sink terms because it was assumed that $c_{3}\left(\mathrm{Cl}^{-}\right)$and $c_{4}$ $\left(\mathrm{Na}^{+}\right)$does not take part in any reactions. This is not true if a salt film can be formed. The effect of salt film formation will be studied in a future study.

The electrostatic potential, $\varphi$, is governed by Eq. (21) coupled with the governing Eqs. (18) and (24-28). The diffusivity $D_{\mathrm{i}}$ is a function of the order parameter $\eta$. As it is known, the diffusivity of ionic species differs in the metal and electrolyte phase. The diffusivities of all the ions were defined using a step function of the order parameter $\eta$. For metal ion $c_{1}$, a step function as expressed in Eq. (30) is used in which the diffusivity value in metal is assumed to be $\gamma$ times less than that in electrolyte. A step function as expressed in Eq. (31) is used for all other ionic species $\left(c_{2}, c_{3}, c_{4}, c_{5}\right.$, and $\left.c_{6}\right)$.

$\mathrm{D}_{1}(\eta)= \begin{cases}D_{1} ; & \eta<0.90 \\ & 0.90 \leq \eta \leq 0.95 \text { (linearly change from } D_{1} \text { to } D_{1} / \gamma \text { ) } \\ D_{1} / \gamma ; & \eta>0.95\end{cases}$

$D_{i}(\eta)= \begin{cases}D_{i} ; \quad & \eta<0.90 \\ \left.0.90 \leq \eta \leq 0.95 \text { (linearly change from } D_{i} \text { to } 0\right) & \\ 0 ; & \eta>0.95\end{cases}$

for $i=2,3, \ldots, 6$.

Overpotential

The overpotential is expressed as

$\varphi_{s, o}=\varphi_{m}-\varphi_{m, s e}-\varphi_{c}-\varphi_{l}$

where $\varphi_{\mathrm{m}}$ is the potential in the metal phase also known as applied potential; $\varphi_{\mathrm{m}, \mathrm{se}}$ is the standard electrode potential in the metal; and $\varphi_{c}$ is the concentration overpotential expressed in (33).

$\varphi_{c}=\frac{R_{g} T}{F z_{1}} \ln \frac{c_{1 b}}{c_{e q, l}}$

The concentration of $M_{e}^{z_{1}}$ near the interface is,

$c_{1 b}= \begin{cases}c_{1} ; & (\eta=0.05) \\ 0 ; & (\eta<0.05) \\ 0 ; & (\eta>0.05)\end{cases}$

The electrostatic potential near the interface is,

$\varphi_{l}= \begin{cases}\varphi ; & (\eta=0.05) \\ 0 ; & (\eta<0.05) \\ 0 ; & (\eta>0.05)\end{cases}$

Kinetic interface parameter and overpotential relation

In this model, the metal corrosion is described by the order parameter $\eta$. The corrosion rate is controlled by the kinetic interface parameter $L$. The shift in the corrosion mode from activation-controlled to diffusion-controlled can be modeled by continuous variation of the kinetic interface parameter $L$. The relationship between the kinetic interface parameter $L$ and the corrosion rate is linear in the activation-controlled mode. ${ }^{33}$ From the Butler-Volmer equation, as expressed in (2), the kinetic interface parameter has an effect on overpotential similar to that of the current density, as expressed below in (36). A similar technique is also implemented in a peridynamic model, in which the interface diffusivity is directly related to the current density for Tafel relation. ${ }^{7}$

$L=L_{o}\left[\exp \left(\frac{a_{a} z_{1} F \varphi_{s, o}}{R_{g} T}\right)-\exp \left(-\frac{a_{c} z_{1} F \varphi_{s, o}}{R_{g} T}\right)\right]$

Following the method developed in Refs ${ }^{7}$ and $^{33}$ and using the experimental values for SS304 (reported in Table 1s), $i_{0}=1.0 \times$ $10^{-6} \mathrm{~A} / \mathrm{cm}^{2}$ and $a_{\mathrm{a}}=0.26$, we calculated $L_{0}=1.94 \times 10^{-13} \mathrm{~m}^{3} /(\mathrm{Js})$.

\section{D PF MODEL}

We implemented the PF model to simulate the corrosion evolution in 1D. The simulations are executed at $T=293.15 \mathrm{~K}$ $\left(20^{\circ} \mathrm{C}\right.$ ) with metal potential of $844 \mathrm{mV} \mathrm{SHE}$ (standard hydrogen electrode) (i.e., $600 \mathrm{mV}$ SCE [saturated calomel electrode]) in a $1 \mathrm{M}$ $\mathrm{NaCl}$ solution. The temperature dependence of the diffusion coefficient is governed by the Einstein relation. ${ }^{12}$ The PF simulation results for the corrosion length are then compared 


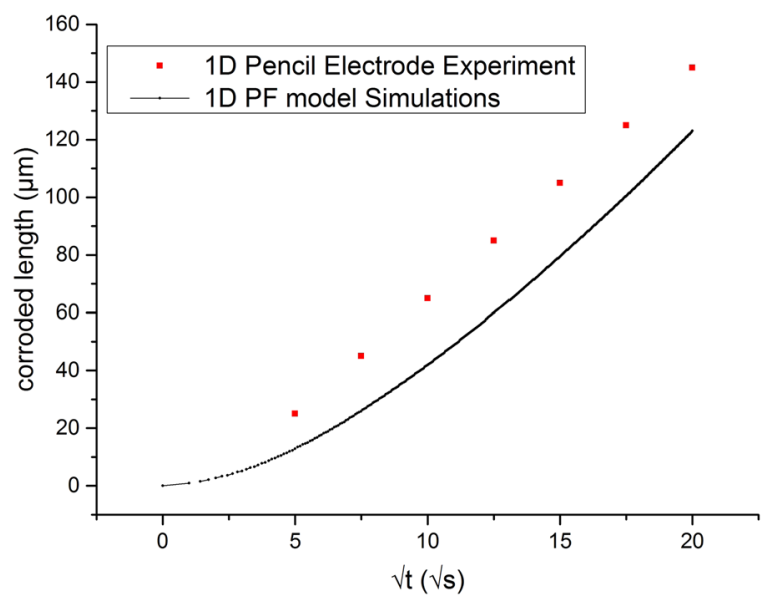

Fig. 2 Comparison of corrosion kinetics of 1D PF model with 1D pencil electrode (1D growth in an artificial pit) in $1 \mathrm{M} \mathrm{NaCl}$ at $20^{\circ} \mathrm{C}$ and $600 \mathrm{mV}(\mathrm{SCE})^{3}$

with the 1D pencil electrode of experimental findings. ${ }^{3}$ The simulations are performed for $400 \mathrm{~s}$, and the results of the corroded length are plotted against the square root of time $(\sqrt{t})$. The simulation results agree well with the experimental results, as illustrated in Fig. 2. The 1D PF model and the 1D pencil electrode experimental results show similar slopes.

A qualitative study on the concentration distribution of ionic species inside the electrolyte is performed as done in many classical numerical models for crevice and pitting corrosion. ${ }^{9,10,48}$ It is difficult to quantitatively measure the molar concentration distribution of ionic species in the electrolyte experimentally. Because such experimental data is lacking, we have discussed these concentration variations theoretically. Figure 1s (supplementary material) shows the concentration in $\mathrm{mol} / \mathrm{L}$ on a logarithmic scale. The higher value of metal ions near the interface results in a slight increase in the $\left[\mathrm{H}^{+}\right]$ion concentration (i.e., a decrease in the $\mathrm{pH}$ value) due to strong coupling between $C_{1}, C_{2}$, and $C_{5}$. The value of $\left[\mathrm{H}^{+}\right]$increases as the overpotential increases because it results in a higher production rate of metal ions and hydrolysis of metal ions. Although, this study was performed on a lower overpotential, but a small increase in $C_{5}$ can still be seen in Fig. 1s (supplementary material). This increase in positive charge is neutralized by the migration of chloride ions towards the interface, as shown in Fig. 1s (supplementary material).

To investigate the effects of metal potential, several simulations were performed to show the behavior of corrosion under different metal potentials. Figure $2 \mathrm{~s}$ (supplementary material) shows that the corrosion rate obtained for these metal potentials are of the same order of the magnitude as the experimental results. ${ }^{49}$ The experimental results plotted in Fig. 2s (supplementary material) give the maximum corrosion rates that can be achieved at the corresponding metal potential. A calibration study was also performed to achieve a corrosion rate for PF 1D model simulation close to experimental ones by varying exchange current density $\left(i_{0}\right)$. It was found that if the value of $i_{0}$ is chosen equal to twice the reported value $\left(i_{0}=1 \times 10^{-6} \mathrm{~A} / \mathrm{cm}^{2}\right)$ in Table $1 \mathrm{~s}$ (supplementary material), then the corrosion rate agree well with the experimental values. ${ }^{49}$ For the sake of consistency, all the presented modeling results are simulated by using the same of value of $i_{0}$ as reported in Table 1s (supplementary material). The overpotential for the corresponding corrosion rates are shown in Fig. 3s (supplementary material).

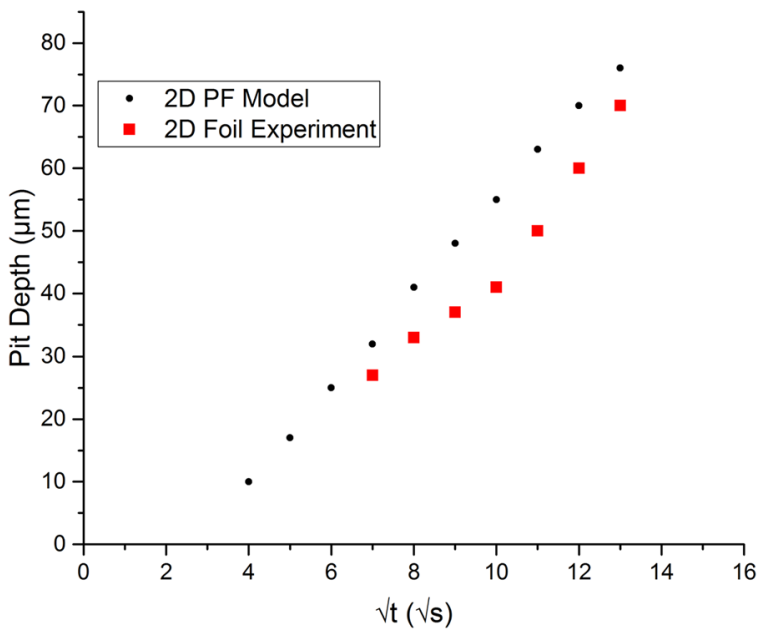

Fig. 3 Comparison of $2 \mathrm{D}$ PF model with $2 \mathrm{D}$ foil experiment at $600 \mathrm{mV}(\mathrm{SCE})$ at $20^{\circ} \mathrm{C}^{3}$

PF simulations for 2D model

The 2D simulations are performed with a metal potential of $600 \mathrm{mV}$ SCE ( $844 \mathrm{mV} \mathrm{SHE})$. The boundary conditions and initial values are the same as described in Fig. 7s (supplementary material). To compare the $2 \mathrm{D} \mathrm{PF}$ model results with the experimental ones, a $300 \mu \mathrm{m}$ by $240 \mu \mathrm{m}$ rectangular geometry is considered in which the metal and electrolyte parts are equally divided, as shown in Fig. 4s (supplementary material). A $60 \mu \mathrm{m}$ wide and $20 \mu \mathrm{m}$ deep semi-elliptical pit is assumed. The remaining surface, as shown in Fig. 4s, is considered to have a passive oxide film. Figure 4s (supplementary material) shows the concentration distribution inside the electrolyte at various time intervals. In Fig. 3 , the 2D PF model results are compared with the 2D foil experiment results reported in the literature. ${ }^{3}$ The evolution of pitting depth over the time shows a trend similar to that found in the experimental results ${ }^{3}$ but with deeper pitting depths than the experimantal data. As mentioned earlier, the regrowth of passive film may be an important factor. We will include the formation of passive film in a future study. The contours of the electrostatic potential distribution for the simulations with the above conditions are shown in Fig. 5s (supplementary material).

\section{Case studies}

Case study 1: Interaction of closely located pits. In reality, multiple pits can nucleate due to changes in chemistry on the metal surface, whereas most numerical models consider only the nucleation or growth of a single pit. A few efforts have been made to understand both experimentally and numerically the interaction of multiple pits. ${ }^{50,51}$ Because we have not considered pit initiation in our PF model, we apply our PF model to two narrow initial openings of $5 \mu \mathrm{m}$ each at distances of (a) $12 \mu \mathrm{m}$ and (b) $5 \mu \mathrm{m}$ at an applied metal potential of $200 \mathrm{mV}$ SHE. The boundary conditions and initial values are the same as those given in Fig. 7s (supplementary material). Figure 4 shows the changes in the morphology of pits with and without interaction in (b) and (a), respectively. It can be seen that without their interaction, these pits corrode at a rate similar to that at which they grow individually. After the pits interact, the chemical compositions of the ionic species change in the vicinities of the pits in the electrolyte. The interaction between the two pits can have either a positive or negative effect on pit growth. ${ }^{52}$ In this study the interaction of the two closely located pits had a positive effect which can be seen in Fig. 4 (b) at $t=6 \mathrm{~s}$. The corroded material in both cases was estimated which suggested that the corrosion rate was increased in case (b). Two pits finally coalesce to form a wider 
(a)
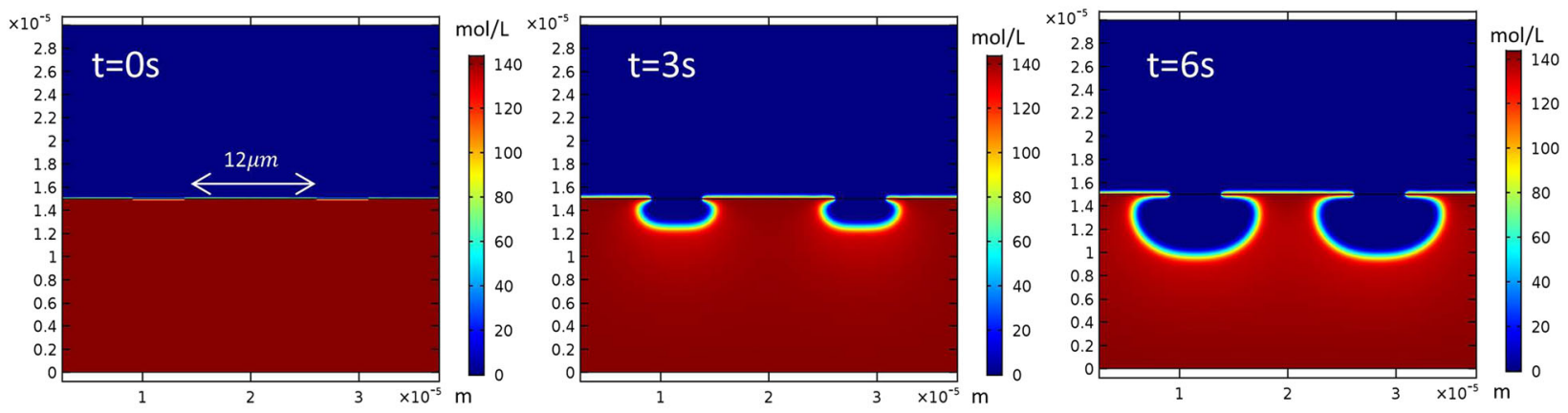

(b)
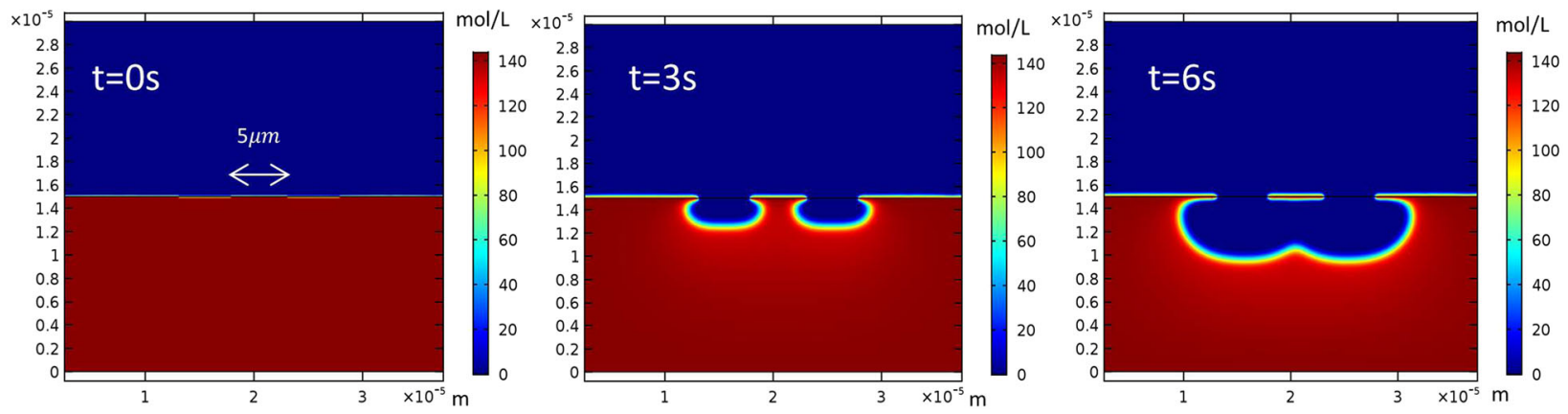

Fig. 4 Multiple-pit morphology changes over time and changes in corrosion rate after the interaction of two pits
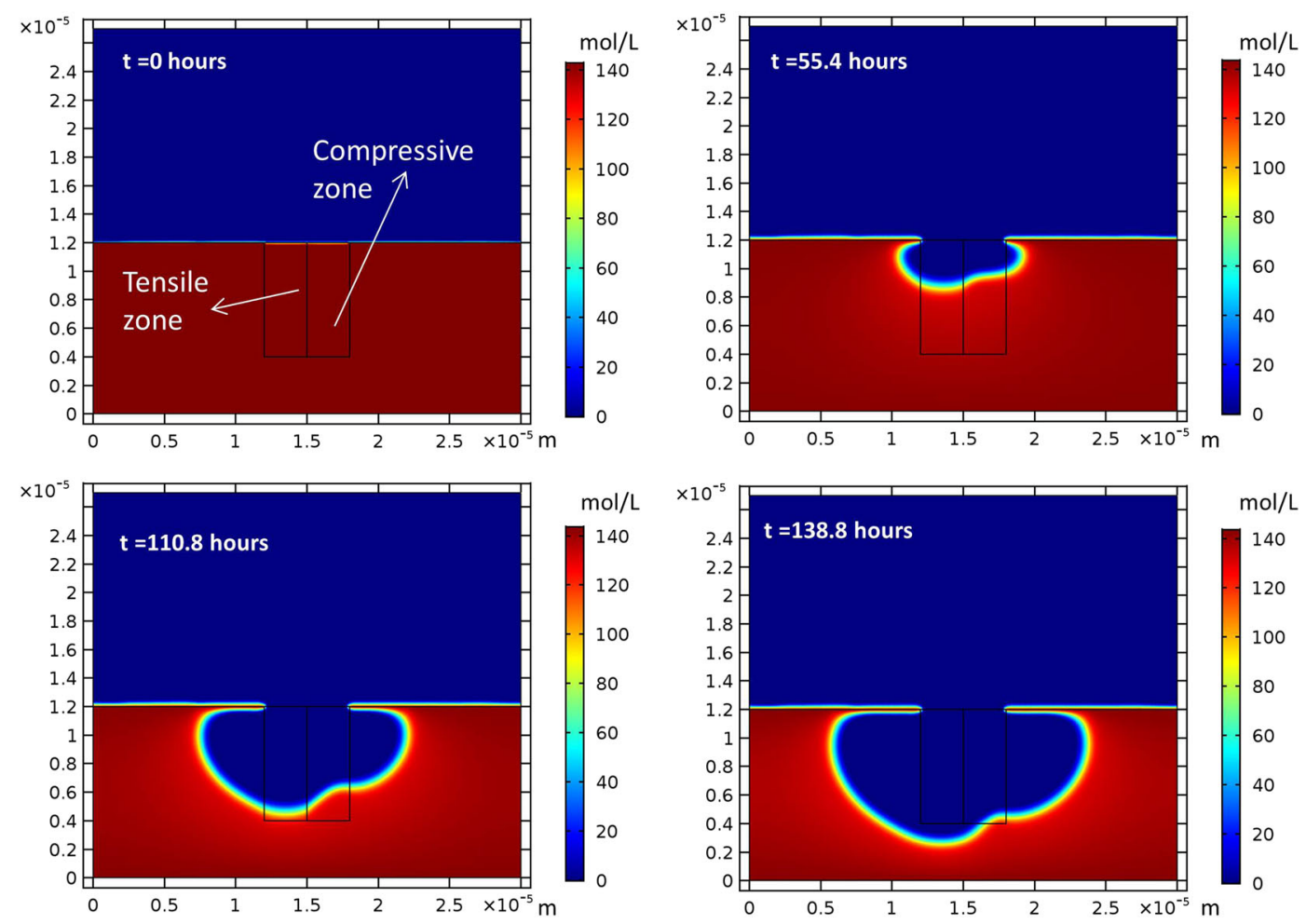

Fig. 5 Pit growth at $\varphi_{m}=-400 \mathrm{mV}$ (SHE) for a material with tensile and compressive residual stresses

pit, similar to the pits formed in real-life metallic structures (i.e., multiple pits nucleate on the corroding surface and interact with each other), which are wider.

Case study 2: Pitting corrosion in a stressed material. Like other alloys, stainless steel can have stressed zones (tensile and compressive). It is believed that overpotential is not uniform in the case of stressed zones, which results in different corrosion rates in different material locations and directions. The experimental finding $s^{53}$ show that most pits grow in locations near the scratched lines on the surface that result from mechanical polishing. These scratched lines could result in strain hardening, as revealed by 

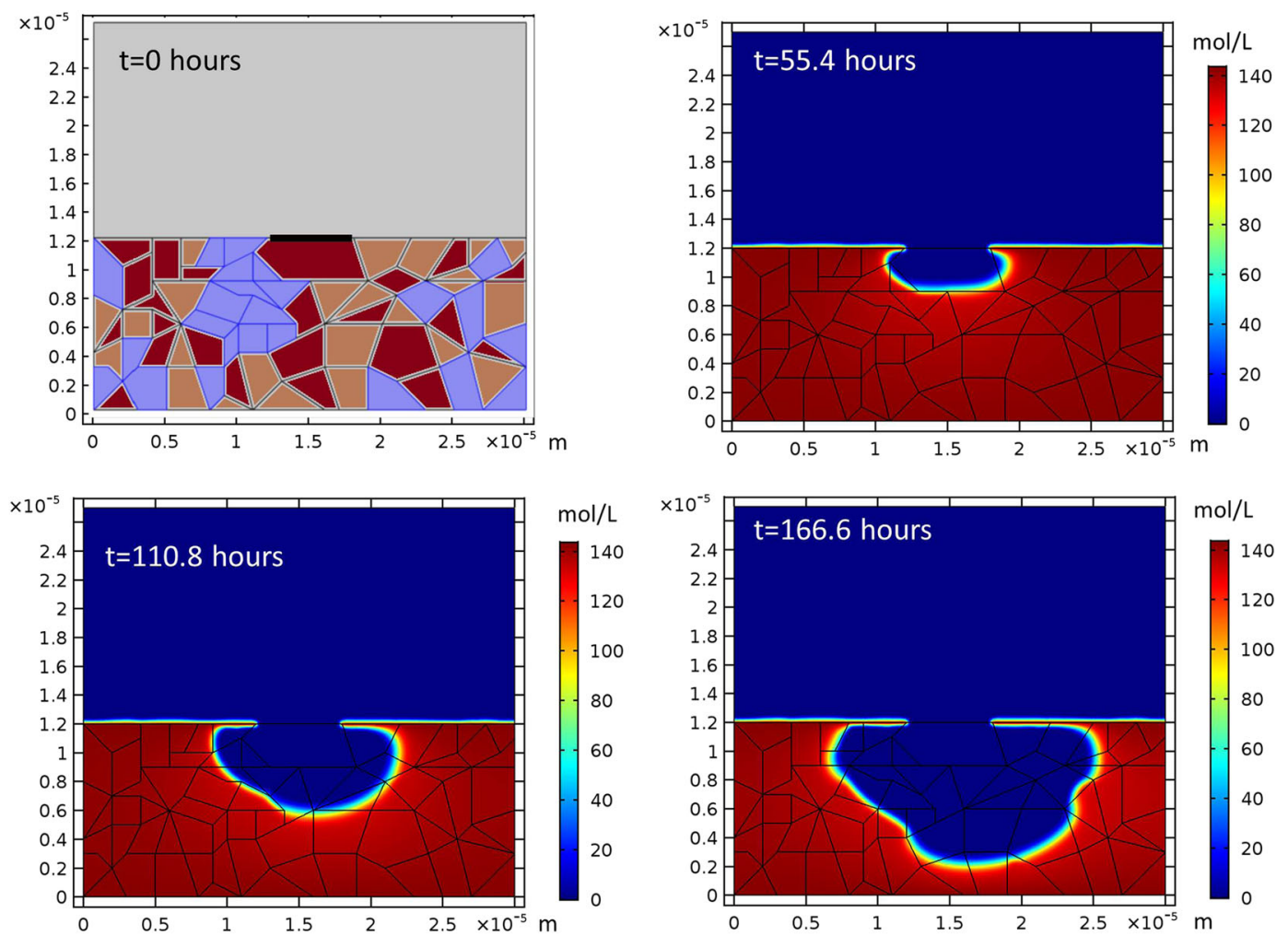

Fig. 6 Pit morphology evolution over time under crystalline dependent corrosion
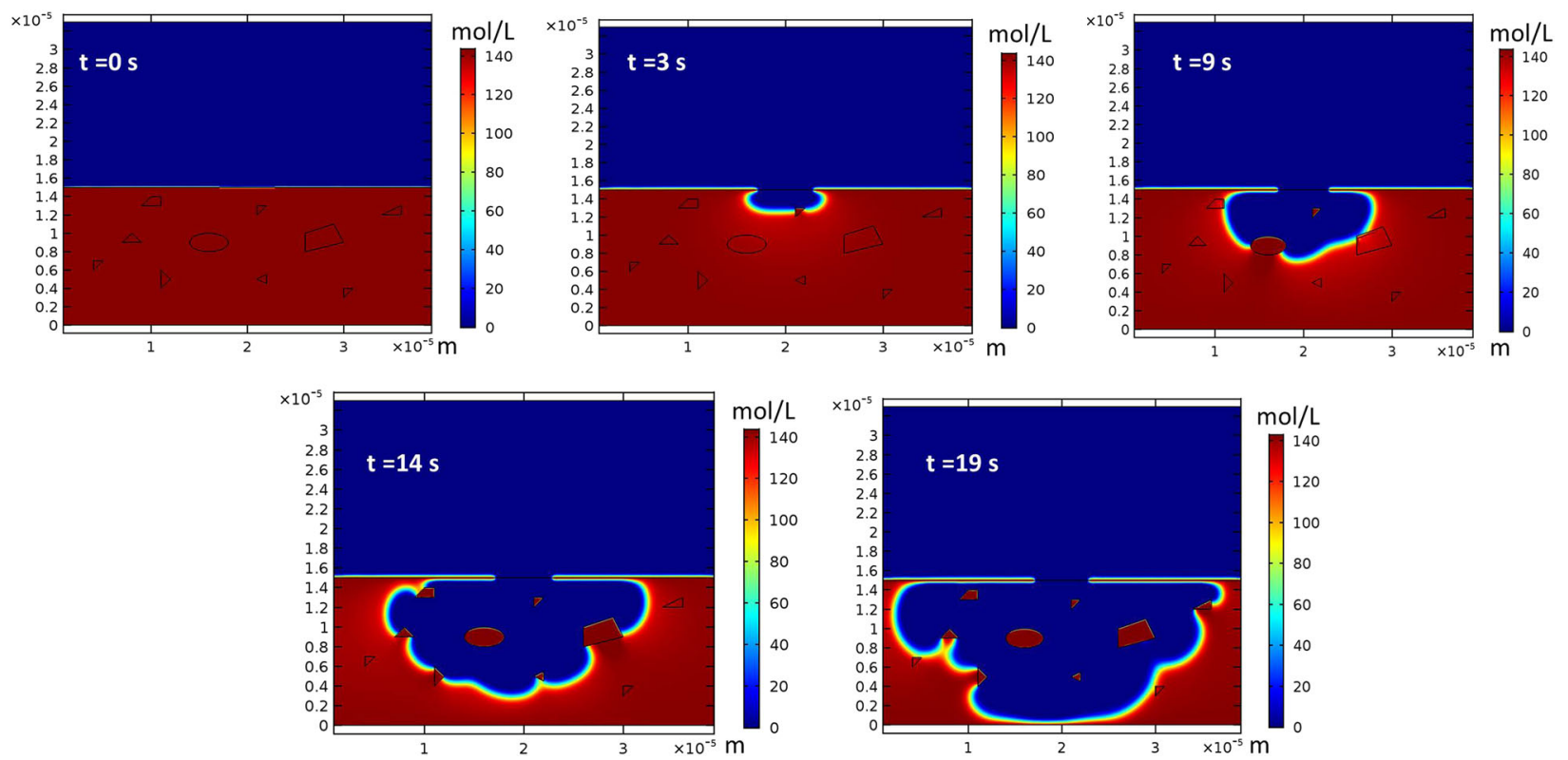

Fig. 7 Pit growth in ceramic particle-reinforced steel at $\varphi_{m}=200 \mathrm{mV}$ (SHE)

electrochemical analysis. ${ }^{53}$ The experimental findings also illustrate that overpotential is not uniform in the presence of residual stresses. Gutman explained the same phenomenon with his theoretical model in which the compressive stress zone has less overpotential than the unstressed zone and the tensile stress zone. ${ }^{54}$ The overpotential is directly related to the corrosion current density. The relationship between the overpotential of the compressive stress zone $\left(\varphi_{\text {comp,s }}\right)$, the unstressed zone $\left(\varphi_{s, 0}\right)$, and the tensile stress zone $\left(\varphi_{\text {tens, } s}\right)$ is $\varphi_{\text {comp }, \mathrm{s}}<\varphi_{s, o}<\varphi_{\text {tens }, \mathrm{s}}$. It should be noted that corrosion rate in plastically deformed zones is greater than that of elastically deformed zones, due to the presence of high density dislocations in plastically deformed zones. In this study, we applied the overpotential dependence on applied/residual stress proposed by Gutman. ${ }^{54}$ According to Gutman's model, ${ }^{54}$ residual stress of $600 \mathrm{MPa}$ corresponds to a change in overpotential of about $20 \mathrm{mV}$ in our system. Here, we model a material with both tensile and compressive stress zones, which have an overpotential difference of $\varphi_{\text {comp }, \mathrm{s}}=\varphi_{\mathrm{s}, \mathrm{o}}-20 \mathrm{mV}$ and $\varphi_{\text {tens,s }}=\varphi_{\mathrm{s}, \mathrm{o}}+20 \mathrm{mV}$, whereas $\varphi_{\mathrm{s}, \mathrm{o}}$ is calculated from (32). A small opening of $6 \mu \mathrm{m}$ is considered at the material's surface. The boundary conditions and initial values are the 
same as those given in Fig. 7s (supplementary material). Figure 5 shows that areas under tensile stress corrode at a faster rate than areas in the compressive stress zone. The pit morphology is closer to that of pits formed during a natural corrosion process because, in most natural scenarios, the corrosion process begins when the passive film is damaged by strain hardening of the surface. In fact, in most of these cases, multiple pits coalesce and grow faster along width than depth. This process is already illustrated in the previous case in which two closely located pits interact.

Case Study 3: Crystallographic plane-dependent pitting corrosion. Several studies suggested that crystallographic orientations greatly affect the propagation rates and morphology of the corroding pits. ${ }^{55-57}$ This dependence is usually attributed to factors such as close packing of crystal planes, reaction rate variation along different plane orientations and density of crystalline defects on micro scale. Here, we demonstrate that this PF model can be a good tool to study this phenomenon in detail. The crystal orientations affect the rate of corrosion because planes with lower atomic densities usually corrode at faster rates than planes with higher atomic densities. ${ }^{57}$ It has been reported that the corrosion rate tends to increase in the order of $\{111\}<\{110\} \leq$ $\{100\}$. The corrosion rate in the crystallographic plane $\{111\}$ is one third of $\{100\}{ }^{57}$ The scenario in which planes $\{110\}$ and $\{100\}$ corrode at the same rate is considered because no exact value is available for their ratio. We implemented our PF model to study the effects of the crystallographic plane orientation on pit growth. The domain geometry considered is $30 \mu \mathrm{m} \times 27 \mu \mathrm{m}$, as shown in Fig. 6. The PF simulations are performed at a lower metal potential of $-400 \mathrm{mV}$ SHE because it is believed that the crystallographic orientation dependence is limited to lower overpotentials when the corrosion process is activation controlled. ${ }^{33,58} \mathrm{~A}$ small opening of $6 \mu \mathrm{m}$ is considered at the surface of the material. The initial values and boundary conditions are the same as described in Fig. $7 \mathrm{~s}$ (supplementary material). Crystallographic planes $\{111\},\{110\}$, and $\{100\}$ are represented by blue, brown, and magenta, respectively, in Fig. 6 , which shows that the pit shape is no longer uniform because $\{111\}$ corrodes at one third the rate of the other two planes. This pit morphology illustrates the strength of our PF model under complex microstructures, with which most sharp interface models fail to cope.

Case Study 4: Pitting corrosion in ceramic particle-reinforced steel. Ceramic particles such as $\mathrm{TiB}_{2}$ and/or $\mathrm{TiC}$ are often embedded into steel to improve its stiffness, strength, ${ }^{59}$ and wear resistance. ${ }^{60}$ Although the addition of these ceramic particles improves some of the material's mechanical properties, it has very little effect on corrosion resistance. ${ }^{61}$ In fact, these reinforcements may enhance stress corrosion cracking (SCC) because they can change the stress distribution near the pits. In case of SCC, a higher stress concentration can be resulted at the tip of a growing pit when the pit reaches a ceramic particle. Metal corrodes faster at the high tensile stress region in the vicinity of a ceramic particle. As we are not studying SCC in this study, the effect of stress concentration will not be considered here. Because the ceramic particles are far less reactive than steel, we assume that they are non-corrodible in salt solution. To ensure that the ceramic particles do not corrode in the salt solution, we considered $L$ to be equal to zero for the ceramic particles. A small opening of $6 \mu \mathrm{m}$ is assumed at the surface of the material. The boundary conditions and initial values are the same as those described in Fig. 7s (supplementary material). Figure 7 shows that the pit morphology changes with the presence of ceramic materials. This example elaborates the ability of this PF model to handle complex structures.

In this study, we have developed a PF model for metal corrosion with ion transport in the electrolyte and this model is used to study pitting corrosion of SS304 in salt water. It is shown that once the kinetic interface parameter is calibrated with the material's exchange current density, the model has the potential to predict corrosion behavior over the whole range of reaction and diffusioncontrolled processes. The simulation results showed that the PF model predictions agree well with the experimental results and that the model has the ability to handle complex microstructures, such as the interaction of closely located pits, the effects of stress on pitting, the effects of ceramic particles, and crystallographic plane orientation on corrosion.

\section{METHOD}

Finite element method, Galerkin method, ${ }^{62}$ is used for space discretization while Backwards differentiation formula (BDF) method $^{63}$ is used for the time integration of the governing partial differential Eqs $(17,18,21,24-28)$. Triangular Lagrangian mesh elements were chosen to discretize the space. It was ensured that we have at least 12 mesh elements inside the diffuse interface to accurately approximate $\eta$ (order parameter) and the piecewise functions based on $\eta$.

Data availability

The data and codes supporting the findings of this study are available from the corresponding author on reasonable request.

\section{ACKNOWLEDGEMENTS}

This work was supported by Research Grants Council of Hong Kong (PolyU 152140/ $14 \mathrm{E})$.

\section{AUTHOR CONTRIBUTIONS}

S.Q.S. conceived the idea, designed and supervised the project. T.Q.A. developed the model, performed simulations and wrote the manuscript. Z.X. and S.Q.S. also contributed in developing the model. S.Q.S., S.Y.H., Y.L. and J.L. provided critical comments and contributed to revisions of the manuscript.

\section{ADDITIONAL INFORMATION}

Supplementary Information accompanies the paper on the npj Computational Materials website (https://doi.org/10.1038/s41524-018-0089-4).

Competing interests: The authors declare no competing interests.

Publisher's note: Springer Nature remains neutral with regard to jurisdictional claims in published maps and institutional affiliations.

Change history: In the original published HTML version of this Article, some of the characters in the equations were not appearing correctly. This has now been corrected in the HTML version.

\section{REFERENCES}

1. Sharland, S. M. A review of the theoretical modelling of crevice and pitting corrosion. Corros. Sci. 27, 289-323 (1987).

2. Ernst, P. \& Newman, R. C. Pit growth studies in stainless steel foils. I. Introduction and pit growth kinetics. Corros. Sci. 44, 927-941 (2002).

3. Ernst, P. \& Newman, R. C. Pit growth studies in stainless steel foils. II. Eff. Temp. Chloride Conc. Sulphate Addit. Corros. Sci. 44, 943-954 (2002).

4. Williams, D. E., Westcott, C. \& Fleischmann, M. Studies of the initiation of pitting corrosion on stainless steels. J. Electroanal. Chem. Interfacial Electrochem. 180, 549-564 (1984).

5. Engelhardt, G. \& Macdonald, D. D. Unification of the deterministic and statistical approaches for predicting localized corrosion damage. I. Theoretical foundation. Corros. Sci. 46, 2755-2780 (2004).

6. Laycock, N. J., Noh, J. S., White, S. P. \& Krouse, D. P. Computer simulation of pitting potential measurements. Corros. Sci. 47, 3140-3177 (2005).

7. Chen, Z. \& Bobaru, F. Peridynamic modeling of pitting corrosion damage. J. Mech. Phys. Solids 78, 352-381 (2015).

8. Duddu, R. Numerical modeling of corrosion pit propagation using the combined extended finite element and level set method. Computational Mechanics 54, 613627 (2014). 
9. Sharland, S. M. \& Tasker, P. W. A mathematical model of crevice and pitting corrosion - I. The physical model. Corros. Sci. 28, 603-620 (1988)

10. Sharland, S. M. A mathematical model of crevice and pitting corrosion - II. The physical model. Corros. Sci. 28, 621-630 (1988).

11. Sarkar, S., Warner, J. E. \& Aquino, W. A numerical framework for the modeling of corrosive dissolution. Corros. Sci. 65, 502-511 (2012).

12. Scheiner, S. \& Hellmich, C. Stable pitting corrosion of stainless steel as diffusioncontrolled dissolution process with a sharp moving electrode boundary. Corros. Sci. 49, 319-346 (2007).

13. Abodi, L. C. et al. Modeling localized aluminum alloy corrosion in chloride solutions under non-equilibrium conditions: steps toward understanding pitting initiation. Electrochim. Acta 63, 169-178 (2012).

14. Galvele, J. Transport processes in passivity breakdown-II. Full hydrolysis of the metal ions. Corros. Sci. 21, 551-579 (1981)

15. Galvele, J. R. Transport processes and the mechanism of pitting of metals. J. Electrochem. Soc. 123, 464-474 (1976).

16. Krawiec, H., Vignal, V. \& Akid, R. Numerical modelling of the electrochemical behaviour of $316 \mathrm{~L}$ stainless steel based upon static and dynamic experimental microcapillary-based techniques. Electrochim. Acta 53, 5252-5259 (2008).

17. Turnbull, A. \& Thomas, J. A model of crack electrochemistry for steels in the active state based on mass transport by diffusion and ion migration. J. Electrochem. Soc. 129, 1412-1422 (1982)

18. Walton, J. C. Mathematical modeling of mass transport and chemical reaction in crevice and pitting corrosion. Corros. Sci. 30, 915-928 (1990).

19. Oldfield, J. W. \& Sutton, W. H. Crevice corrosion of stainless steels: II. Experimental studies. Br. Corros. J. 13, 104-111 (1978).

20. Oldfield, J. W. \& Sutton, W. H. Crevice corrosion of stainless steels: I. A mathematical model. Br. Corros. J. 13, 13-22 (1978).

21. Hebert, K. \& Alkire, R. Dissolved metal species mechanism for initiation of crevice corrosion of aluminum: I. Experimental investigations in chloride solutions. J. Electrochem. Soc. 130, 1001-1007 (1983).

22. Watson, M. K. \& Postlethwaite, J. Numerical simulation of crevice corrosion: the effect of the crevice gap profile. Corros. Sci. 32, 1253-1262 (1991).

23. Sharland, S. M. A mathematical model of the initiation of crevice corrosion in metals. Corros. Sci. 33, 183-201 (1992).

24. Friedly, J. C. \& Rubin, J. Solute transport with multiple equilibrium-controlled or kinetically controlled chemical reactions. Water Resour. Res. 28, 1935-1953 (1992).

25. White, S. P., Weir, G. J. \& Laycock, N. J. Calculating chemical concentrations during the initiation of crevice corrosion. Corros. Sci. 42, 605-629 (2000).

26. Webb, E. G. \& Alkire, R. C. Pit initiation at single sulfide inclusions in stainless steel: III. Mathematical model. J. Electrochem. Soc. 149 (2002).

27. Gavrilov, S., Vankeerberghen, M., Nelissen, G. \& Deconinck, J. Finite element calculation of crack propagation in type 304 stainless steel in diluted sulphuric acid solutions. Corros. Sci. 49, 980-999 (2007).

28. Venkatraman, M. S., Cole, I. S. \& Emmanuel, B. Corrosion under a porous layer: a porous electrode model and its implications for self-repair. Electrochim. Acta 56, 8192-8203 (2011).

29. Xiao, J. \& Chaudhuri, S. Predictive modeling of localized corrosion: an application to aluminum alloys. Electrochim. Acta 56, 5630-5641 (2011).

30. Scheiner, S. \& Hellmich, C. Finite volume model for diffusion- and activationcontrolled pitting corrosion of stainless steel. Comput. Methods Appl. Mech. Eng. 198, 2898-2910 (2009).

31. Onishi, Y., Takiyasu, J., Amaya, K., Yakuwa, H. \& Hayabusa, K. Numerical method for time-dependent localized corrosion analysis with moving boundaries by combining the finite volume method and voxel method. Corros. Sci. 63, 210-224 (2012).

32. Li, Y., Hu, S., Sun, X. \& Stan, M. A review: applications of the phase field method in predicting microstructure and property evolution of irradiated nuclear materials. npj Comput. Mater. 3, 16 (2017).

33. Mai, W., Soghrati, S. \& Buchheit, R. G. A phase field model for simulating the pitting corrosion. Corros. Sci. 110, 157-166 (2016).

34. Mai, W. \& Soghrati, S. A phase field model for simulating the stress corrosion cracking initiated from pits. Corros. Sci. 125, 87-98 (2017).

35. Kim, S. G., Kim, W. T. \& Suzuki, T. Phase-field model for binary alloys. Phys. Rev. E 60, 7186-7197 (1999).

36. Chen, L. Q. Phase-field models for microstructure evolution. Annu. Rev. Mater. Res. 32, 113-140 (2002)

37. Moelans, N., Blanpain, B. \& Wollants, P. An introduction to phase-field modeling of microstructure evolution. Calphad 32, 268-294 (2008).

38. Guo, X. H., Shi, S.-Q. \& Ma, X. Q. Elastoplastic phase field model for microstructure evolution. Appl. Phys. Lett. 87, 221910 (2005).

39. Guo, X. H., Shi, S. Q., Zhang, Q. M. \& Ma, X. Q. An elastoplastic phase-field model for the evolution of hydride precipitation in zirconium. Part I: smooth specimen. J. Nucl. Mater. 378, 110-119 (2008).
40. Guo, X. H., Shi, S. Q., Zhang, Q. M. \& Ma, X. Q. An elastoplastic phase-field mode for the evolution of hydride precipitation in zirconium. Part II: specimen with flaws. J. Nucl. Mater. 378, 120-125 (2008).

41. Wheeler, A. A., Boettinger, W. J. \& McFadden, G. B. Phase-field model for isothermal phase transitions in binary alloys. Phys. Rev. A 45, 7424-7439 (1992).

42. Hu, S. Y., Murray, J., Weiland, H., Liu, Z. K. \& Chen, L. Q. Thermodynamic description and growth kinetics of stoichiometric precipitates in the phase-field approach. Calphad 31, 303-312 (2007).

43. Ginzburg, V. L. On the theory of superconductivity. II Nuovo Cim. (1955-1965) 2, 1234-1250 (1955).

44. Cahn, J. W. \& Hilliard, J. E. Free energy of a nonuniform system. I. Interfacial free energy. J. Chem. Phys. 28, 258-267 (1958).

45. Pongsaksawad, W., Powell, A. C. \& Dussault, D. Phase-field modeling of transportlimited electrolysis in solid and liquid states. J. Electrochem. Soc. 154, F122-F133 (2007).

46. Guyer, J. E., Boettinger, W. J., Warren, J. A. \& McFadden, G. B. Phase field modeling of electrochemistry. II. Kinet. Phys. Rev. E 69, 021604 (2004).

47. Leblanc, P., Cabaleiro, J., Paillat, T. \& Touchard, G. Impact of the laminar flow on the electrical double layer development. J. Electrost. 88, 76-80 (2017).

48. Turnbull, A. \& Ferriss, D. Mathematical modelling of the electrochemistry in corrosion fatigue cracks in structural steel cathodically protected in sea water Corros. Sci. 26, 601-628 (1986).

49. Revie, R. W. \& Uhlig, H. H. Uhlig's Corrosion Handbook, 3rd edn (Wiley, Hoboken, New Jersey, 2011).

50. Budiansky, N. D., Organ, L., Hudson, J. L. \& Scully, J. R. Detection of interactions among localized pitting sites on stainless steel using spatial statistics. J. Electrochem. Soc. 152, B152-B160 (2005).

51. Laycock, N., White, S. \& Krouse, D. Numerical simulation of pittingcorrosion: interactions between pits in potentiostatic conditions. ECS Trans. 1, 37-45 (2006).

52. Laycock, N. J., Krouse, D. P., Hendy, S. C. \& Williams, D. E. Computer simulation of pitting corrosion of stainless steels. Electrochem. Soc. Interface 23, 65-71 (2014).

53. Martin, F. A., Bataillon, C. \& Cousty, J. In situ AFM detection of pit onset location on a 304L stainless steel. Corros. Sci. 50, 84-92 (2008).

54. Gutman, E. M. Mechanochemistry of Solid Surfaces. (World Scientific Publishing Company, Singapore, 1994).

55. Shahryari, A., Szpunar, J. A. \& Omanovic, S. The influence of crystallographic orientation distribution on 316LVM stainless steel pitting behavior. Corros. Sci. 51, 677-682 (2009).

56. Kumar, B. R., Singh, R., Mahato, B., De, P. K., Bandyopadhyay, N. R. \& Bhattacharya, D. K. Effect of texture on corrosion behavior of AISI 304L stainless steel. Mater. Charact. 54, 141-147 (2005).

57. Lindell, D. \& Pettersson, R. Crystallographic effects in corrosion of austenitic stainless steel 316L. Mater. Corros. 66, 727-732 (2015).

58. Frankel, G. S. Pitting corrosion of metals: a review of the critical factors. J. Electrochem. Soc. 145, 2186-2198 (1998).

59. Akhtar, F. Ceramic reinforced high modulus steel composites: processing, microstructure and properties. Can. Metall. Q. 53, 253-263 (2014).

60. Akhtar, F. Microstructure evolution and wear properties of in situ synthesized TiB2 and TiC reinforced steel matrix composites. J. Alloy. Compd. 459, 491-497 (2008).

61. Pagounis, E. \& Lindroos, V. K. Processing and properties of particulate reinforced steel matrix composites. Mater. Sci. Eng. A 246, 221-234 (1998).

62. Fairweather, G. Finite Element Galerkin Methods for Differential Equations, Lecture Notes in Pure and Applied Mathematics, vol. 34, Marcel Dekker, New York, (1978).

63. Ascher, U. M. \& Petzold, L. R. Computer Methods for Ordinary Differential Equations and Differential-Algebraic Equations. Vol. 61 (Society For Industrial Applied Mathematics, U.S., Philadelphia, 1998).

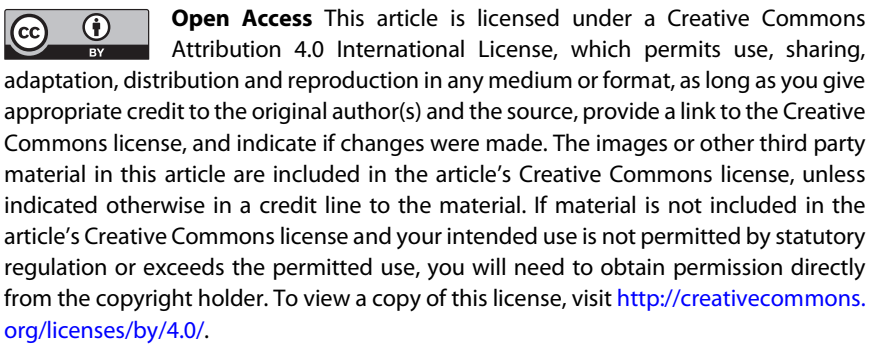

C) The Author(s) 2018 